\title{
Ability of the ischaemic myocardium to absorb blood perfused artificially through a previously implanted artery ${ }^{1}$
}

\author{
PANAGIOTIS C. PETROPOULOS² \\ From the Department of Cardiovascular Surgery, Presbyterian Medical Center, San Francisco, California
}

Blood absorption from intramyocardially implanted arteries has been evaluated both experimentally and clinically (Vineberg and Jewett, 1947 ; Glenn and Beal, 1950 ; Bencosme and Vineberg, 1953 ; Bakst, Maniglia, Adam, and Bailey, 1955; Sabiston, Fauteux, and Blalock, 1957; Haeger and Wallgren, 1959a, b ; and Lopez-Belio, Rodriguez, Stansel, Tasaki, Gomez-Ferrer, and Julian, 1961). Middle calibre arteries, such as the carotid or subclavian, have been implanted into the myocardium, not only with their ends open but even with side holes in the embedded portion, without the formation of a haematoma. Such implanted arteries have been found to stay patent, usually for four to six weeks. Some such implantations have remained patent for up to 246 days, though similar arterial implants into skeletal muscle or other organs, such as liver and spleen, produce early haematomas with resulting obliteration of the lumen of the implanted arteries (Sabiston et al., 1957). Haeger and Wallgren (1959b) proved that plastic tubes transplanted into the myocardium, bridging the internal mammary artery and the left ventricular wall, could be kept patent for about six weeks, which was long enough to supply the myocardium with oxygenated blood during the critical phase of ligation of the coronary artery. When similar shunts with tubes were used in the same manner in various organs, such as the liver, spleen, and muscles, they became obstructed after two to five days. Direct grafting of a homologous vessel or a prosthetic device from the aorta to the myocardium has been experimentally and clinically applied (Smith, Beasley, Hodes, Hall, Biel, and Huth, 1957; Haeger and Wallgren, 1959b).

\footnotetext{
I Supported by grants from the American Heart Association and the U.S. Public Health Service.

2 Fellow in cardiovascular surgery, Cora and Webb Mading of Surgery, Baylor University College of Medicine, Houston, Texas, Stanford University Medical School, Palo Alto, California, and the Institute of Medical Sciences, Presbyterian Medical Center, San Francisco, California.
}

The blood coming from the lumen of the arterial implants naturally does not maintain its initial intra-arterial pressure. We could not find any reports about the pressure of this extra-arterial blood which inundates the myocardium. We suggested that the pressure of this blood might be greater than that of the extravascular blood perfusing the myocardium at low pressure and less than the intra-arterial pressure.

The possibility of absorption by the ischaemic myocardium of blood perfused at systemic or higher pressures through arteries previously implanted into the myocardium has been studied in the following experiments.

\section{METHODS}

In 20 mongrel dogs, each weighing from 17 to $22.5 \mathrm{~kg}$. and anaesthetized with intravenous nembutal, cyclic positive pressure insufflation of the lungs was maintained with a mixture of $50 \%$ oxygen and air. The left thorax was opened through the fifth interspace. The left internal mammary artery was prepared from the second to the sixth intercostal spaces and transected at the level of the seventh rib. The last $6 \mathrm{~cm}$. of the central part of the artery, together with its bleeding intercostal branches, was then embedded in a tunnel dissected through the complete length of the left ventricular myocardium, 2 to $3 \mathrm{~mm}$. beneath the epicardium and about $2 \mathrm{~cm}$. to the left of the anterior descending coronary artery. The pulling of the artery through the tunnel was done very gently and great care was taken not to twist its axis. The open end was fixed with two 6-0 sutures to the surrounding tissues in such a way that it remained open. Kinking of the entrance of the tunnel did not occur. Before closing the orifices of the tunnel the undisturbed flow through the artery was checked.

The anterior descending coronary artery was temporarily occluded distal to the septal artery, 
and retropressures were obtained about 15 seconds later as above. A magnesium-aluminium-alloy wire (Gage, Olson, and Chardack, 1956) was inserted into the artery using the same technique. The pericardium was closed with two sutures.

In five of these dogs, the expected pericardial and pulmonic adhesions to the ischaemic myocardium were avoided by isolating the anterior surface of the left ventricle with a nylon sheet (Petropoulos, in preparation) $0.5 \mathrm{~mm}$. thick, fixed to the internal surface of the anterior pericardium (Fig. 1). In five additional control dogs of about the same weight the pericardium was left widely open after insertion of the wire, and the chest was closed.

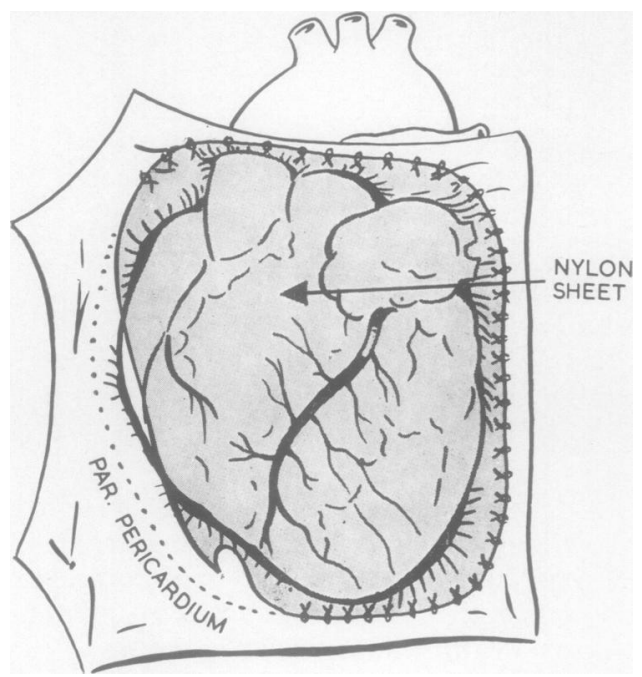

FIG. 1. A nylon sheet isolates the anterior surface of the left ventricle.

\section{RESULTS}

All five dogs with the protective nylon sheet died within eight days. Of the remaining 15 dogs with mammary artery implantation, 10 died and five survived till the predicted time of sacrifice (100 days). Of the five control dogs, three died within ten days and two survived. Pathological investigation of the dead animals has shown that patency of the implanted mammary artery was evident in two dogs with the protective sheet $(50 \%$ patency of the lumen) and in six without the sheet (30 to $50 \%$ patency of the lumen of the intramyocardial portion). The myocardium tolerated the implanted vessel well. There was little fibrosis in the immediate vicinity of the vessel and no haematoma. The upper third of the anterior descending coronary artery with the metallic implant was occluded in all the animals (Fig. 2). Soft pericardial adhesions were present when the nylon sheet was not used. Transmural infarctions were present in

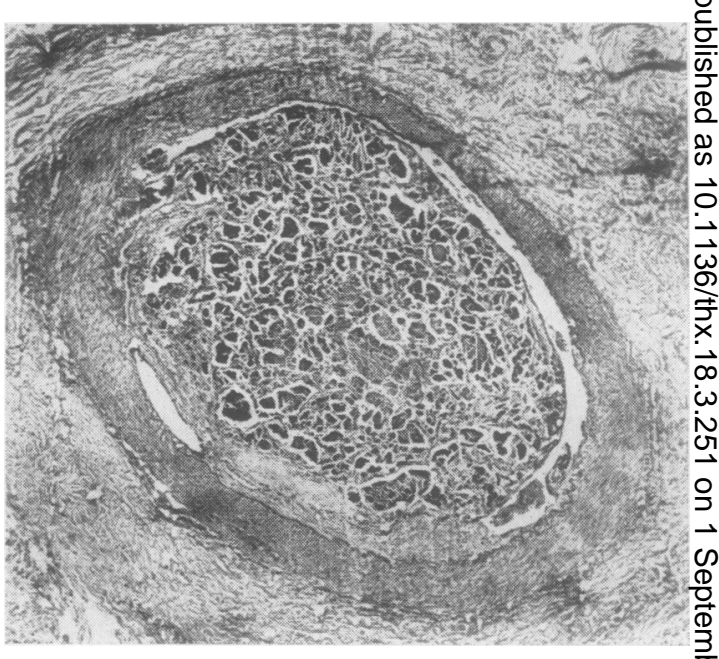

FIG. 2. Complete disintegration of the magnesiumaluminium-alloy wire one month after its insertion into the lumen of the anterior descending coronary artery.

four dogs with protective nylon sheets and in three without. In the rest of the animals the infarction waso subendocardial. No significant differences as to the extension of the infarctions were found in the two 0 groups. The dead dogs of the control group hado only subendocardial infarctions and soft pericardial $\stackrel{\Phi}{\complement}$ adhesions.

In the surviving animals the chest was re-opened 100 days after the initial operation. Fibrous pericardial and pulmonic adhesions were present, embedding the extracardial segment of the mammary artery which was patent in four animals. The retropressure of the intramural portion of the mammary artery was between $62 / 5$ and $75 / 8 \mathrm{~mm}$. Hg. The back-flow was $x$ $0 \cdot 2,0.3,0 \cdot 1,0.2$, and $0.2 \mathrm{ml}$. per minute during theo first three minutes and then stopped completely.

The upper segment of the anterior descending coronary artery was occluded. The retropressure of 3 the patent distal segment was $74 / 62,60 / 41,58 / 40$, 0 $52 / 38$, and $57 / 45 \mathrm{~mm}$. Hg. The back-flow of the anterior descending coronary artery was $1 \cdot 2,0 \cdot 7,1 \cdot 4$, 0.9 , and $1.9 \mathrm{ml}$. per minute. When the implanted internal mammary artery was clamped, the coronary back-flow remained unchanged.

The distal portion of the clamped internal mammary 0 artery was cannulated and perfused with saturated N heparinized blood at a rate of 30 drops $(2 \mathrm{ml}$.) per $\sigma$ minute and at a pressure of between 180 and $300 \mathrm{~mm}$. Hg. We had to increase continually the perfusione pressure in order to keep the perfusing rate constant. $\mathbb{D}$ After 5 minutes' perfusion no more blood could be ${ }^{+}$ perfused. The back-flow and back-pressure of the anterior descending coronary artery remained un-웅 changed during, and for 25 minutes after, the per- $\odot$ fusion (Fig. 3). The animals were then sacrificed. The $\mathbb{\mathbb { D }}$ intramural portion of the implanted internal mammary artery was patent (in three animals $80 \%$ in its middle 


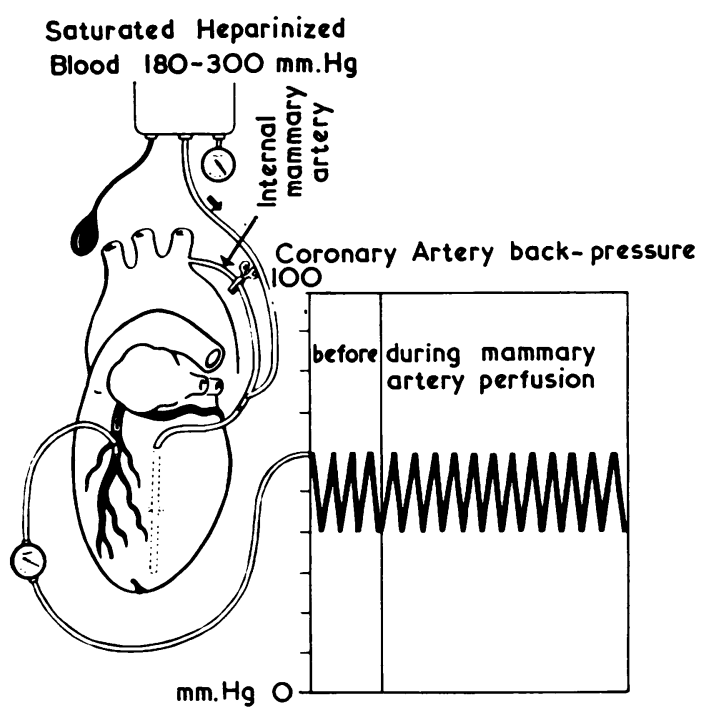

Saturated Heparinized

Blood $180-300 \mathrm{~mm} . \mathrm{Hg}$

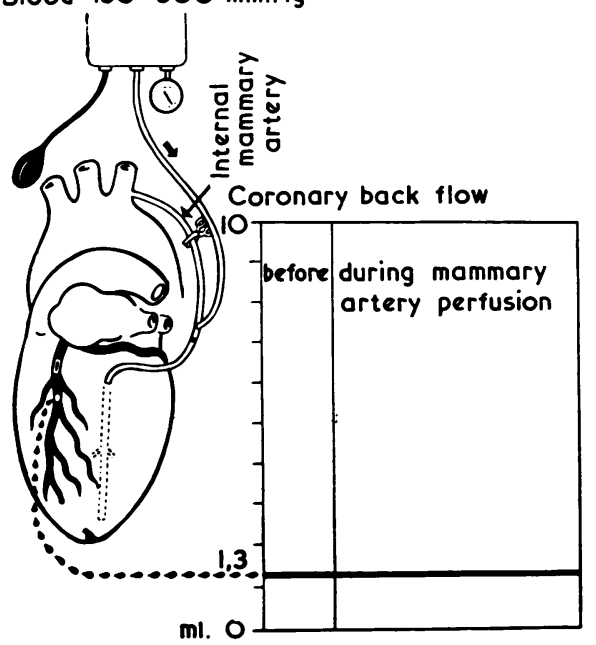

FIG. 3. Back-pressure and back-flow of the anterior descending coronary artery during perfusion with saturated blood at a pressure of between 180 and $300 \mathrm{~mm} . \mathrm{Hg}$ to the distal portion of the intramyocardially transplanted internal mammary artery.

and distal portion, and in two animals $20 \%$ patent). Evidence of healed subendocardial infarctions was present in all five surviving dogs.

The two surviving control dogs were sacrificed on the hundredth post-operative day. The central segment of the anterior coronary artery was thrombosed. The retropressure of the distal part was $66 / 41$ and $70 / 48$ $\mathrm{mm}$. $\mathrm{Hg}$. The back-flow was 1.2 and $1.1 \mathrm{ml}$. per minute. Healed subendocardial infarctions were found on section not significantly different in size from those in the survivors after internal mammary artery implantation.

\section{DISCUSSION}

In these experiments the mortality rate was $100 \%$ when the ischaemic myocardium perfused from a mammary artery was isolated by a nylon sheet from the surrounding tissues. The mortality rates of the dogs with unprotected hearts and of the control animals with simple pericardiotomy were similar. The back-pressures and back-flows of the occluded coronary arteries of the hearts with a patent mammary artery implant, and of the controls, were the same. The back-flow of the implanted artery was minimal and soon stopped. The back-pressure of the mammary artery corresponds to the intramyocardial pressure at this myocardial level. Intima proliferation of the distal intramyocardial segment of the mammary artery was present in all surviving dogs. A reaction of the myocardium to the implanted artery or haematoma formation was not found. The extent of the subendocardial infarction in the surviving and in the control dogs was about the same.

We did not perform these experiments in order to study the fate of the implanted mammary artery. This question has already been studied by other investigators. The purpose of our experiments was to study two questions.

(1) We examined the changes of back-flow and back-pressure in the anterior descending coronary artery, when the distal part of the previously implanted patent internal mammary artery had been artificially perfused. The results were expected to prove the possibility of revascularization using this procedure. In the literature we could find no information regarding this. Data are given only about the anterior descending back-flow changes when the mammary transplant is temporarily clamped (Sabiston et al., 1957). We performed many experiments before we could obtain a patent mammary artery 100 days after the implantation. Perfusion of this artery did not change the backflow level of the coronary artery.

The changes in coronary sinus flow during the perfusion have not been investigated because it is known that only a fraction of the left coronary artery blood returns to the general venous circulation through the coronary sinus, the remaining blood discharging into the cardiac cavities via the anterior cardiac veins and the thebessian vessels.

Some investigators injected radio-opaque materials directly into the distal portion of the implanted mammary artery. When they found 
these dyes in coronary vessels at some distance from the implanted artery, they came to the erroneous conclusion that the newly formed vessels communicated with the transplanted artery. In some additional experiments of our own on the beating or non-beating freshly excised heart we showed that after a rapid injection of $5 \mathrm{ml}$. of a $2 \%$ solution of Indian ink in water, or of dextran at a pressure of $180 \mathrm{~mm} . \mathrm{Hg}$, the dye readily filled the coronary veins and arteries of the neighbouring region and was macroscopically visible (Petropoulos, in preparation). If fluorescein was injected intramyocardially the coronary circulation of the dog became fluorescent immediately after the injection of the dye. Conversely, if the same amount of blood, containing $2 \%$ Indian ink dye or fluorescein, was injected in the same way, and in the same region, the dye did not visibly fill the regional vessels. It is probable that the high viscosity of the blood was the cause of this failure to fill the regional larger vessels quickly. Thorotrast, a low viscosity radio-opaque dye, injected intramyocardially also filled the surrounding vessels (Fig. 4). We conclude that the perfusion of dye through the transplanted artery under pressure has the same distribution as intra-myocardially injected dye and that it does not demonstrate the vascular connexion of the graft through any newly formed vessels.

(2) The absolute mortality after isolation of the grafted ischaemic heart from the surrounding tissues by a nylon sheet, excluding every possibility of revascularization through adhesions, proves also the possibility of vascularization of the implanted internal mammary artery ex juvandibus.

\section{SUMMARY}

Implantation of the left internal mammary artery into the ischaemic left ventricle in 20 dogs resulted in a mortality rate of $67 \%$ when adhesions to the ischaemic myocardium were formed, and $100 \%$ when adhesions were prevented from forming by the interposition of a nylon sheet. The maximal patency of the lumen of the intramyocardial portion of the implanted artery 100 days after the implantation was $80 \%$ of its diameter. When the implanted mammary artery was clamped or perfused at systemic or higher pressure the back-flow and back-pressure of the anterior descending coronary artery remained unchanged.

\section{REFERENCES}

Bnkst, A. A., Maniglia, R., Adam, A., and Bailey, C. P. (1955). The physiologic and pathologic evaluation of the implantation of the internal mammary artery into the left ventricular myocardium for the treatment of coronary artery disease. Surgery, 38, 349.

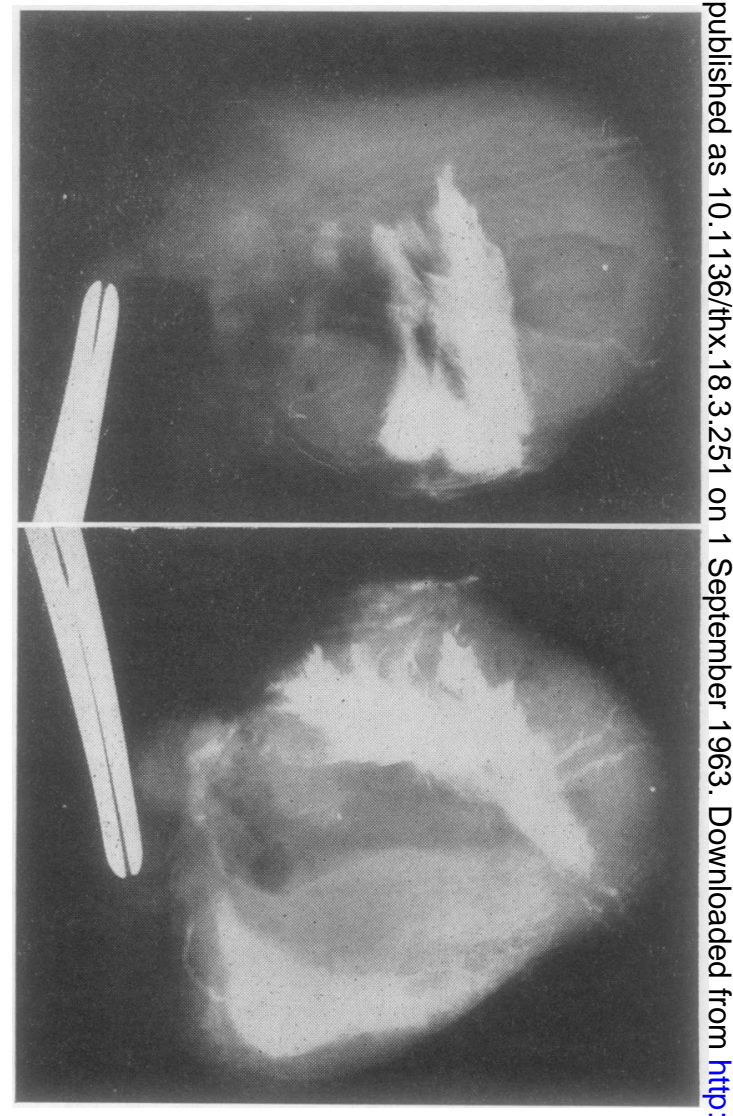

FIG. 4. Linear areas of density having the appearance of contrast material in collateral coronary vessels extending in several directions laterally from the mean concentration. of the injected contrast material to the left ventricular myocardium.

Bencosme, S. A., and Vineberg, A. (1953). Histologic studies of the internal mammary artery after implantation into the myocardium. 음

Gage, A. A., Olson, K. C., and Chardack, W. M. (1956). Description of a method. Ann. Surg., 143, 535 .

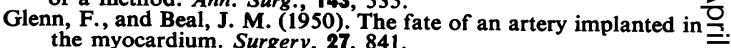
the myocardium. Surgery, 27, 841 .

Haeger, K., and Wallgren, G. R. (1959a). Studies of the coronary $N$ circulation. III. Intramyocardial implantation of nylon tubes. after ligation of a major coronary artery. J. thorac. Surg., 37, 532. N (1959b). Studies of the coronary circulation. IV. Surgical treatment of acute experimental myocardial infarction in the dog. $\mathrm{N}$
Ibid., 37, 545.

Lopez-Belio, M., Rodriguez, S., Stansel, H. C., Tasaki, G., GomezFerrer, F., and Julian, O. C. (1961). Effects of continuous flowthrough implanted mammary artery and myocardial ischemia once mammary-coronary communications. $J$. thorac. cardiovasc. $\frac{\mathrm{C}}{\mathrm{D}}$
Surg., 42, 648.

Sabiston, D. C., Fauteux, J. P., and Blalock, A. (1957). An experi- $\mathscr{\mathscr { C }}$ mental study of the fate of arterial implants in the left ventricular myocardium, with a comparison of similar implants in other $\square$ organs. Ann. Surg a comparist

Smith, S., Beasley, M., Hodes, R., Hall, H., Biel, E., and Huth, E. W. (1957). Auxiliary myocardial vascularization by prosthetic graft implantation. Surg. Gynec. Obstet., 104, 263.

Vineberg, A. M., and Jewett, B. L. (1947). Development of an anastomosis between the coronary vessels and a transplanted internal mammary artery. Canad. med. Ass. J., 56, 609. 